INRA Prod. Anim., 1996, 9 (1), 35-44

\section{M. de REVIERS}

INRA Station de Recherches Avicoles 37380 Nouzilly

\title{
Photopériodisme, développement testiculaire et production de spermatozoïdes chez les oiseaux domestiques
}

On sait depuis longtemps piloter la production d'œufs au moyen de programmes d'éclairement mis en œuvre dans des bâtiments conditionnés. Peut-on en faire autant pour la production de spermatozoïdes chez les différentes espèces utilisées en aviculture? Cette question est d'autant plus importante que l'usage de l'insémination artificielle, maintenant de règle chez des espèces économiquement aussi importantes que la Dinde ou les Palmipèdes, fait percevoir bien mieux qu'en reproduction naturelle les déficiences éventuelles de la reproduction mâle. Celles-ci, amplifiées par la sélection sur la vitesse de croissance et l'hypertrophie des masses musculaires pectorales, ont pu être en grande partie surmontées à temps grâce à la recherche de méthodes d'élevage orientées vers la maîtrise de la production de spermatozoïdes.

Chez les Oiseaux sauvages et deux espèces domestiques (le Canard Pékin et la Caille japonaise), c'est surtout le mâle qui a servi de modèle pour mettre en évidence le rôle de la lumière dans les variations saisonnières du développement des gonades. Ce rôle y est en

\section{Résumé}

La reproduction du Coq peut être dans une large mesure contrôlée par la durée quotidienne d'éclairement. Ce sont les variations de cette durée qui permettent le mieux de maîtriser la précocité de la production de spermatozoïdes, tandis que la persistance de cette production peut, sauf cas particulier, être la plus satisfaisante en jours courts.

Cette condition est donc contradictoire à la fois avec une production de spermatozoïdes précoce, qui nécessite des jours croissants, et avec le maintien d'un intensité de ponte élevée, car on ne sait l'obtenir qu'en jours longs.

Comme palliatif, on utilise donc dans la pratique un large effectif de coqs dont l'alimentation est par ailleurs rationnée afin de maintenir leur libido. L'insémination artificielle serait sans doute plus satisfaisante, en permettant d'adopter des conditions d'élevage spécifiques des coqs tout en réduisant leur nombre au minimum. Largement employée dans les autres espèces avicoles, son utilisation pour produire des poussins de chair bute encore sur des habitudes, sur la nécessité d'employer plus de personnel, plus qualifié et plus motivé, et sur une structure de filière où l'éleveur est encore parfois client de l'accouveur. effet plus marqué que chez les femelles des espèces sauvages, où l'intervention de stimuli autres que l'éclairement peut être indispensable au développement complet des ovaires et à la ponte.

Les recherches sur le contrôle photopériodique du développement testiculaire ont été très tôt orientées vers la connaissance des voies et des mécanismes d'action de la lumière. Chez les Oiseaux, l'étude des variations du développement testiculaire est restée au stade d'un simple bilan pondéral, sans étude quantitative précise de la spermatogenèse. Or c'est le plus ou moins bon déroulement de celle-ci qui conditionne les variations de la production de spermatozoïdes, composante essentielle de la fertilité mâle.

Dans une logique de production animale, l'important est de déterminer si cette fertilité peut être contrôlée, voire améliorée, et dans quelle mesure la maîtrise de l'éclairement est un moyen d'y parvenir. Ce facteur du milieu peut être facilement contrôlé dans la pratique, puisque la production avicole industrielle se fait en bâtiments conditionnés. 
Il est important de souligner ici le poids économique de la reproduction chez les Oiseaux domestiques. Mis à part le cas des poules pondeuses (production nationale d'œufs : 15 milliards d'unités/an, soit 900000 t), cette reproduction est en France à l'origine de près de 1 milliard 300 millions de poussins destinés, après élevage, à la production de viande, d'un total national de 1850000 t/an (dont Poulet: $50 \%$, Dindon : $27 \%$, Canard de Barbarie : 8 $\%$, Pintade : $3 \%$ ).

Ces poussins sont issus de croisements de lignées très sélectionnées en faveur de la vitesse de croissance, du développement des muscles pectoraux et d'un faible engraissement, spécialisation qui se serait accompagnée d'une diminution des performances de reproduction, en particulier du côté mâle, si une stratégie de recherche n'avait pas été développée dans le même temps pour contrecarrer cette tendance.

Il nous a semblé important que cette stratégie porte à la fois sur la mise au point de méthodes de reproduction (insémination artificielle : cf notamment Brillard et de Reviers 1989), et sur celle de méthodes d'élevage. Parmi ces dernières, les programmes d'alimentation (traités par ailleurs : de Reviers 1990) ont un rôle primordial, mais les aspects concernant l'éclairement sont, comme nous allons le voir, tout aussi importants, et font donc l'objet du présent article. cette période peuvent donc avoir des répercussions importantes sur la précocité sexuelle et le potentiel de reproduction.

\section{1 / Comment caractériser les effets de l'éclairement sur la reproduction mâle?}

Les approches de ce problème sont très différentes de celles utilisables chez les femelles.

Chez ces dernières, il est admis que le stock de follicules primordiaux est entièrement constitué au moment de l'éclosion et s'épuise peu à peu au cours de la vie post-natale, soit par atrésie, soit du fait des ovulations successives.

Dans le cas du mâle, le nombre des lignées germinales, loin d'être fixé au moment de l'éclosion, augmente considérablement pendant les 4 à 6 premiers mois de la vie postnatale. Les conditions d'élevage subies pendant la croissance peuvent donc avoir des répercussions importantes non seulement sur la précocité sexuelle, comme chez la Poule, mais encore sur le potentiel de reproduction des adultes.

De plus, il y a renouvellement des spermatogonies souches jusqu'à un âge avancé : ainsi se trouvent assurées la continuité et la pérennité de la production de spermatozoïdes. Cela n'exclut pas qu'il y ait des variations importantes de leur nombre en fonction de l'âge, car ce renouvellement et les étapes suivantes de la spermatogenèse fonctionnent avec un rendement variable.
Par ailleurs, si les effets de l'éclairement sur la ponte peuvent être caractérisés par des critères externes simples, faciles et rapides à mesurer (par exemple l'âge au premier œuf, l'évolution de l'intensité de ponte, etc), chez le mâle, la mesure de la production de gamètes est plus difficile à réaliser. Il ne suffit pas, en effet, de récolter le sperme et d'y dénombrer les spermatozoïdes pour caractériser leur production par les testicules à un instant donné. Nous avons montré qu'il faut en réalité que les récoltes de sperme soient faites à une fréquence et pendant une durée suffisantes à chaque âge étudié, pour qu'elles puissent refléter, ne fût-ce que grossièrement, le niveau de la production testiculaire de spermatozoïdes. Ce type de mesure demande beaucoup plus de temps et de qualification que le dénombrement des œufs pondus. Il reste très dépendant du savoir-faire technique et des réactions comportementales des mâles. Dans ces conditions, on peut connaître le nombre des spermatozoïdes disponibles pour l'insémination, mais on ne peut rien savoir du rendement numérique de la spermatogenèse à ses différentes étapes.

Or la spermatogenèse doit être quantifiée pour que son plus ou moins bon déroulement soit connu. Le nombre des spermatogonies souches est en effet très variable chez le mâle adulte. De plus la spermatogenèse consiste non seulement en une différenciation cellulaire, mais encore en une prolifération cellulaire, grâce aux divisions spermatogoniales et méiotiques, prolifération dont le rendement numérique peut varier considérablement. Dans l'état actuel des connaissances, la spermatogenèse des Oiseaux ne peut être quantifiée qu'à partir du début de la prophase méiotique.

Pour y parvenir, il faut prélever les testicules (ce qui n'est possible qu'une seule fois dans la vie de l'animal !), et dénombrer les cellules germinales de chaque catégorie. $\mathrm{Si}$ on connaît leurs durées de vie respectives, que nous avons déterminées par marquage isotopique et autoradiographie, il est alors possible d'en calculer la production quotidienne, ce que nous avons fait chez le Coq. Les techniques correspondantes peuvent être précises ; elles demandent un gros effort de standardisation, sont très lourdes à mettre en œuvre (histologie quantitative) et très consommatrices de temps. Cela a découragé beaucoup de chercheurs.

Par ailleurs l'évaluation de la fécondance des spermatozoïdes est hérissée de difficultés :

- ou bien elle est très lourde, si elle passe par l'insémination artificielle, car celle-ci doit impliquer de nombreuses femelles et être faite en conditions très standardisées,

- ou bien elle n'est pas assez pertinente, les méthodes in vitro ne permettant vraiment que l'élimination des moins bons éjaculats.

Quand l'objectif est de maîtriser la reproduction mâle, on ne peut se contenter de la simple étude du développement pondéral des testicules faite chez les Oiseaux sauvages ou le Canard Pékin. Plusieurs questions se 
posent, auxquelles il n'est possible de répondre que par des expériences à long terme :

1. Quelles sont les relations entre le développement testiculaire pondéral, l'établissement de la spermatogenèse chez le jeune et les nombres de spermatozoïdes produits pendant la vie adulte ? L'objectif ici est de déterminer à partir de quel âge et pendant combien de temps le mâle est effectivement utilisable comme reproducteur dans les conditions, exigeantes, de l'industrie avicole.

2. Dans quelle mesure est-il possible de contrôler la précocité, le niveau maximum et la persistance de cette production par l'éclairement ? Autrement dit, s'agit-il d'un moyen efficace de contrôler la reproduction mâle?

3. Quelles sont les conséquences d'une plus ou moins grande précocité du développement testiculaire sur la production ultérieure de spermatozoïdes ? Le niveau de cette production à maturité sexuelle est-il lui-même en relation avec la persistance de cette production ? En d'autres termes, est-il possible, en vue de la sélection, de raccourcir l'intervalle entre générations, ou, plus généralement, de diminuer le coût de la période d'élevage, grâce à des mâles précoces ? Si on les obtient, quels sont les effets induits sur le niveau de fertilité des adultes et la persistance ultérieure de ce niveau?

Les réponses à ces deux dernières questions ont une valeur d'application différente suivant le système de reproduction. En reproduction naturelle (utilisée pour la production du poulet de chair), mâles et femelles cohabitent, et subissent donc les mêmes conditions d'éclairement. Ils peuvent au contraire être soumis à des programmes d'éclairement différents, si nécessaire, dans le cas de l'insémination artificielle (utilisée soit en sélection dans toutes les espèces avicoles, soit au niveau des croisements terminaux chez le Dindon, la Pintade et de plus en plus chez les Palmipèdes), car rien n'oblige alors à faire cohabiter les reproducteurs des deux sexes.

\section{2 / Développement des testicules, spermatogenèse et production de spermatozoïdes}

$\mathrm{Au}$ moment de l'éclosion, chez le Coq, les testicules, très petits (moyenne : 2 à $5 \mathrm{mg}$ par testicule), sont déjà organisés en tubes séminifères, constitués d'une membrane basale périphérique, et d'un épithélium comprenant d'une part des cellules somatiques, les cellules de Sertoli, nombreuses mais peu différenciées, et d'autre part des cellules germinales, bien moins abondantes. Les plus évoluées peuvent être des spermatogonies (Coqs de type chair) ou des spermatocytes primaires (Coqs de type ponte, plus précoces).
Pendant la période post-natale, le développement des testicules se poursuit en 3 phases, suivant une courbe pondérale d'allure sigmoïdale (figure 1a). Il est important d'en connaître les caractéristiques, car ce sont les modifications de durée et d'amplitude de ces phases qui peuvent traduire l'action du photopériodisme sur la reproduction mâle.

La première phase (dite prépubère) est caractérisée par l'absence de production de spermatozoïdes et une testostéronémie très faible, bien qu'il y ait sur les 6 premières semaines de vie, augmentation transitoire de la teneur plasmatique en hormone gonadotrope LH.

La spermatogenèse est alors limitée à la production de spermatocytes primaires, de plus en plus nombreux, mais qui dégénèrent en cours de prophase méiotique. Dans le même temps, les cellules de Sertoli commencent par proliférer, puis se différencient progressivement, avec augmentation de leur taille cytoplasmique, et cessent de se multiplier. Il en résulte une augmentation très importante du diamètre et de la longueur totale des tubes séminifères, qui occupent la quasi-totalité $(85$ à $95 \%)$ du volume testiculaire en fin de phase prépubère.

L'ensemble de cette évolution fait que la croissance pondérale des testicules est lente en valeur absolue pendant la phase prépubère, celle-ci se terminant à un poids testiculaire moyen de l'ordre de $500 \mathrm{mg}$ à $1 \mathrm{~g}$. Cela correspond à un âge moyen de 10 à 16 semaines, suivant qu'il s'agit de coqs de type ponte ou chair respectivement, et suivant les conditions de milieu.

Considérée en valeur relative, cette phase de croissance est au contraire très rapide puisque le poids testiculaire est en moyenne plus que centuplé pendant cette période!

La phase suivante, dite période pubère, démarre par une augmentation rapide et soutenue de la teneur plasmatique en $\mathrm{LH}$, suivie immédiatement d'une augmentation de testostéronémie. Les premiers spermatozoïdes, produits dès le début de cette phase, sont de mauvaise qualité et par conséquent peu fécondants (Saeki 1963).

L'accroissement simultané du nombre des lignées germinales fonctionnelles et du rendement des différentes étapes de la spermatogenèse fait que la croissance pondérale absolue des testicules est d'abord très rapide pendant cette période, atteignant 150 à $600 \mathrm{mg} / \mathrm{j}$. Puis elle ralentit, et cesse vers 20 ou 24 semaines d'âge suivant le type de coqs. En coordonnées semi-logarithmiques (figure 1b), il apparaît que cette croissance est assimilable à une exponentielle dans sa première moitié, et qu'elle est ensuite fortement freinée.

$\mathrm{Si}$ on considère cette phase de croissance en valeur relative, on s'aperçoit qu'elle est plus lente que pendant la période prépubère, puisque le poids des testicules n'est multiplié que par 10 à 30 pendant la dizaine de semaines que dure la période pubère, au lieu de 100 et plus pendant la période prépubère.

\author{
Pendant la \\ période post- \\ natale, le \\ développement des \\ testicules présente \\ 3 phases, suivant \\ une courbe \\ pondérale d'allure \\ sigmoïdale. La \\ phase pubère \\ démarre à l'âge de \\ 10 à 16 semaines.
}


Figure 1. a - Développement pondéral des testicules chez le Coq. Les âges et les poids testiculaires correspondant aux différentes périodes (prépubère, pubère, adulte) sont variables suivant l'origine génétique et le milieu (éclairement, alimentation).

$b$ - Relation entre le logarithme du poids testiculaire et l'âge. Sous 16 h d'éclairement et jusqu'à l'âge de 16 semaines, la relation est de la forme : $\log P(t)=\log P_{0}+K t$, soit $P(t)=P_{0} \exp (K t)$, avec $K=0,028$ (log décimaux) ou 0,064 (log népériens).
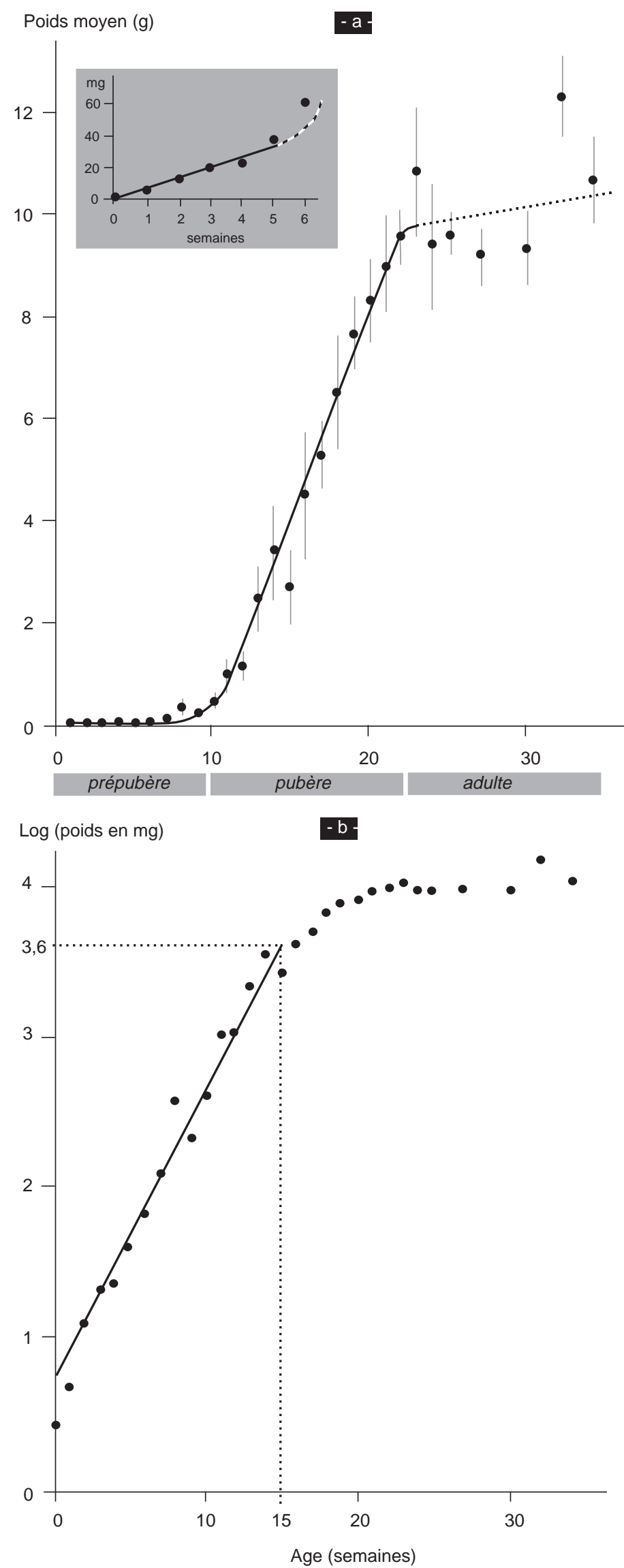

La maturité sexuelle du coq ne correspond pas à un événement bien tranché comme la ponte du premier œuf chez la poule. En effet, le nombre et la qualité des spermatozoïdes produits augmentent progressivement pendant la phase pubère, de sorte que la maturité sexuelle est atteinte peu avant que le nombre de spermatozoïdes produits soit maximum.

Pendant la période adulte, il est souhaitable que l'activité spermatogénétique soit maintenue à un niveau élevé. En effet, les coqs reproducteurs de type chair mis en place en reproduction naturelle avec les poules, sont censés les féconder depuis l'entrée en ponte (âge correspondant : 24 semaines ou moins) jusqu'à l'âge de leur réforme (64 semaines environ). Les poules, et par conséquent les coqs, sont soumis pendant ce temps à des jours longs, réputés favorables au maintien d'une bonne ponte.

Nous allons voir que ce type d'éclairement est au contraire défavorable au maintien d'une activité spermatogénétique élevée chez le Coq, comme chez des Oiseaux sauvages. Précisons que les résultats qui suivent ont été obtenus chez des coqs de type ponte (M519) ou de type chair (I99 et I77), car il peut exister à l'intérieur de cette espèce des réponses photopériodiques différentes chez d'autres types génétiques. Elles varient encore plus dans d'autres espèces avicoles, comme par exemple la Pintade.

\section{3 / Réponses aux photopériodes quotidiennes constantes}

\section{1 / Cas des Oiseaux sauvages}

Chez Zonotrichia leucophrys gambelii (Moineau couronné) ou Junco hyemalis (Junco ardoisé), très étudiés, respectivement par Farner, Wolfson et Follett, il est connu depuis longtemps que le développement testiculaire ne peut se produire qu'en jours de $9 \mathrm{~h}$ ou plus.

Ces auteurs ont proposé de modéliser le développement pondéral des testicules par une exponentielle du temps :

$$
\begin{gathered}
\mathrm{P}(\mathrm{t})=\mathrm{P}_{0} \exp (\mathrm{Kt}) \\
\text { soit } \log (\mathrm{P}(\mathrm{t}))=\log \left(\mathrm{P}_{0}\right)+\mathrm{Kt}
\end{gathered}
$$

où $\mathrm{P}_{0}$ est le poids initial des testicules et $\mathrm{K}$ un coefficient, fonction de l'espèce et de la durée quotidienne d'éclairement $\mathrm{L}$ en heures. Mais cette expression ne décrit bien, comme chez le Coq, que le début de la phase rapide du développement testiculaire, et ne permet pas de connaître le niveau final de ce développement, non plus que son évolution chez l'adulte. 
Pourtant ce sont les variations du coefficient $K$ de cette relation qui ont servi classiquement de référence pour traduire l'efficacité d'un traitement lumineux, voire hormonal, sur le développement testiculaire des Oiseaux sauvages. Il y a en effet une relation quantitative précise (retrouvée par ailleurs chez la Caille domestique) entre la vitesse du développement testiculaire et la durée L de la photopériode quotidienne :

$\mathrm{K}=0,009$ (L - 9,1) (chez Zonotrichia, Farner 1958)

soit $\mathrm{K}=0,063$ pour $\mathrm{L}=16 \mathrm{~h}$, ce qui est du même ordre que la valeur observée chez le Coq (figure 1b).

Cette référence ne concerne donc que des expériences à court terme. On sait par ailleurs qu'en jours longs, le poids testiculaire passe par un maximum, puis décroît irrévocablement, même si la durée des jours est allongée, car l'animal est alors photoréfractaire. Cet état ne peut être levé que par une période de plusieurs semaines de jours courts.

\subsection{Le Coq}

Cette espèce, au contraire des Oiseaux sauvages, produit des spermatozoïdes toute l'année quand elle est soumise aux conditions climatiques naturelles, avec un maximum au printemps et un minimum en été-automne.

Selon Nalbandov (1967) le développement testiculaire du Coq est peu sensible aux variations de la durée quotidienne d'éclairement. En effet, il peut avoir lieu dans la quasi-obscurité (30 minutes d'éclairement par jour). Mais, dans ces conditions, le développement testiculaire est très retardé, ce qui est incompatible en pratique avec une bonne reproduction ! D'autres études avaient été faites, mais dans des conditions trop peu précises pour pouvoir être retenues.

Nous avons montré que chez le Coq soumis depuis le très jeune âge à des photopériodes quotidiennes constantes de 8 ou 16 h, l'âge moyen auquel démarre la phase pubère du développement testiculaire (16 semaines) est peu affecté par la durée d'éclairement. Pendant cette phase, les jours longs induisent une vitesse de croissance testiculaire 2 fois plus élevée qu'en jours courts, de sorte que la maturité sexuelle est atteinte 4 semaines plus tôt. La croissance testiculaire se termine à un poids un peu moindre dans ce dernier cas (16 g/testicule, au lieu de 20, figure 2).

C'est surtout pendant la phase adulte qu'apparaît une grande différence d'évolution du poids testiculaire en fonction de la durée d'éclairement : il régresse en quelques semaines sous les jours longs, qu'ils soient de 16 ou de $12 \mathrm{~h}$ alors qu'il reste stable en moyenne pendant plusieurs mois en jours courts (figure 2).

Les variations de poids testiculaire en fonction de la durée d'éclairement journalière reflètent, pour l'essentiel, celles de l'activité spermatogénétique. A poids testiculaire égal, et à maturité sexuelle, celle-ci est cependant de 15 à $25 \%$ plus élevée sous les jours longs, si bien que le nombre total de spermatozoïdes produits par testicule est de $40 \%$ supérieure à celui observé en jours courts.

Il s'agit bien d'effets spécifiques de l'éclairement, les coqs des deux lots ayant des poids corporels pratiquement égaux en moyenne et en variance à chacun des âges considérés.

La cohabitation des coqs et des poules en jours longs explique donc sans doute en grande partie pourquoi il y a eu pendant longtemps décroissance du taux de fécondation des œufs bien avant la fin de la période de ponte, défaut qui a depuis été corrigé par de meilleures conditions d'élevage.

On a donc pour le Coq une situation différente de celle observée chez les Oiseaux sau-

Figure 2. Développement testiculaire du Coq et activité spermatogénétique sous photopériode constante.

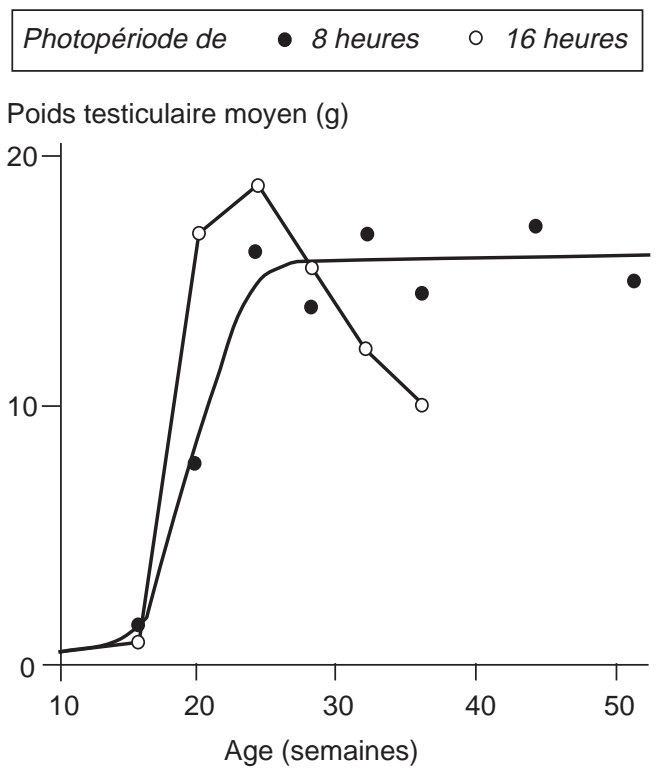

Nombres totaux de cellules germinales (x $10^{9}$ )

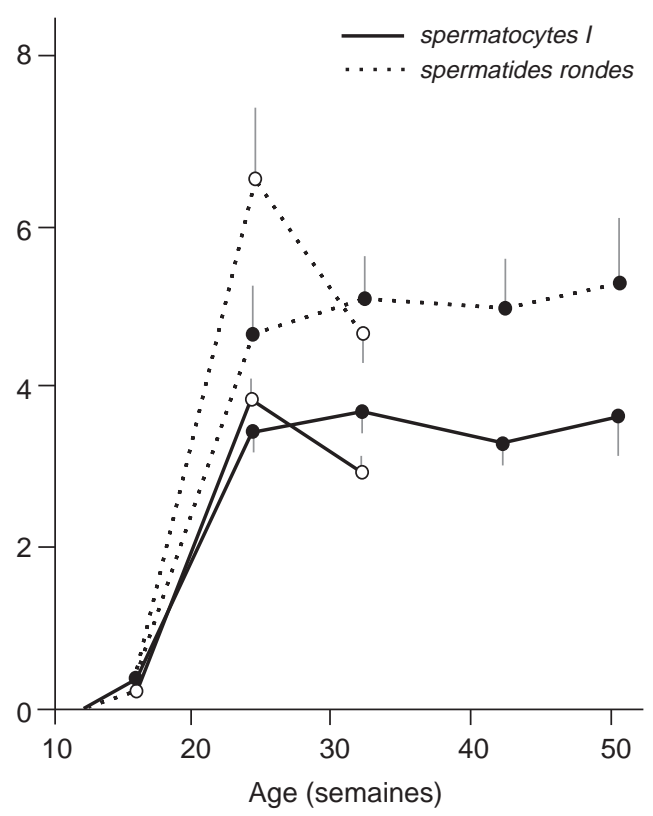

Par rapport à des jours courts, les jours longs doublent la vitesse de croissance des testicules et avancent la maturité sexuelle. Mais le poids final est plus faible. 
vages car le développement testiculaire y est possible en jours de moins de 9 heures. Par contre le poids testiculaire régresse chez les coqs adultes maintenus sous des jours longs, même quand la durée d'éclairement est augmentée de 16 à 20 h. Nous avons eu l'occasion d'observer qu'il peut y avoir, chez certains individus, recrudescence spontanée de la production de spermatozoïdes en jours de $16 \mathrm{~h}$. Il ne s'agirait donc peut-être pas d'un état photoréfractaire stricto sensu, mais d'un simple feed-back des hormones gonadiques, levé après deux à trois mois de régression partielle des testicules.

Par ailleurs, chez le Junco ou l'Etourneau, le maintien d'un développement testiculaire maximum est possible pendant près d'une année et plus en les maintenant respectivement sous $12 \mathrm{~h}$ d'éclairement quotidien. Cette durée occasionne encore la régression testiculaire chez le Coq, où il faut des jours plus courts $(8 \mathrm{~h})$ pour que le poids testiculaire soit maintenu.

depuis l'éclosion à des jours décroissants comparés à des jours croissants, mais le passage à des jours longs à 24 semaines d'âge conduit à un poids testiculaire plus élevé.

\section{4 / Effets des variations de la durée quotidienne d'éclairement}

Les auteurs ayant expérimenté sur Oiseaux sauvages se sont le plus souvent limités à des durées quotidiennes d'éclairement constantes, sans doute pour une plus grande commodité d'interprétation. Il n'en reste pas moins que, dans la nature et sous nos latitudes, la durée quotidienne d'éclairement ne cesse de varier ! On sait qu'elle passe de 8 à $16 \mathrm{~h} \mathrm{du}$ solstice d'hiver à celui d'été, puis de 16 à $8 \mathrm{~h}$ au solstice d'hiver suivant. Chez la Poule, ce sont ces variations, et non la durée d'éclairement par elle-même, qui induisent des âges différents d'entrée en ponte (elle est avancée par les jours croissants, retardée par les jours décroissants).

Nous avons montré que de même, chez le Coq en croissance, les variations de la durée quotidienne d'éclairement ont une influence considérable sur l'âge auquel démarre la phase pubère du développement testiculaire (figure 3).

Ainsi des coqs d'origines génétiques très différentes (croisement M 519 Rhode x Wyandotte x Marans, ou I 99 à base de Cornish) subissent un retard important du développement testiculaire s'ils sont soumis, pendant les 6 mois qui suivent l'éclosion, à des jours décroissant progressivement de 16 à $8 \mathrm{~h}$ (-20 $\mathrm{min} / \mathrm{sem})$. Mais la phase pubère se déclenche tout de même à partir de 20 semaines d'âge, alors que les jours continuent de décroître pendant encore 4 semaines. Leur rôle est donc celui d'un simple freinage.

Si ces mêmes coqs sont passés en jours croissants à partir de 24 semaines d'âge, leurs testicules atteignent en 8 semaines un niveau de développement très élevé, comme s'il y avait effet de rebond. Nous avons vérifié que ces résultats pondéraux sont en bonne concordance avec ceux concernant l'activité spermatogénétique.

A l'opposé la phase pubère est avancée à 12 semaines d'âge (soit avec 8 semaines d'avance sur les jours décroissants, et 4 sur les jours

Figure 3. Développement pondéral des testicules en fonction des variations de la photopériode. a - Chez des coqs soumis depuis l'éclosion à des jours décroissants de 16 à $8 \mathrm{~h}(-20 \mathrm{~min} / \mathrm{sem})$ ou à des jours croissant de 8 à $16 \mathrm{~h}(+20 \mathrm{~min} / \mathrm{sem})$. A l'âge 24 semaines, les coqs soumis aux jours croissants sont maintenus sous une photopériode de $16 \mathrm{~h} / \mathrm{j}$, et les coqs en jours décroissants sont soumis durant 8 semaines à une augmentation de photopériode de $1 \mathrm{~h} / \mathrm{sem}$ puis maintenus à $16 \mathrm{~h}$ de lumière par jour.

$b$ - Développement pondéral des testicules chez des coqs soumis à des jours croissant rapidement (+ 1 h/sem) à partir de 8, 14 ou 20 semaines d'âge.
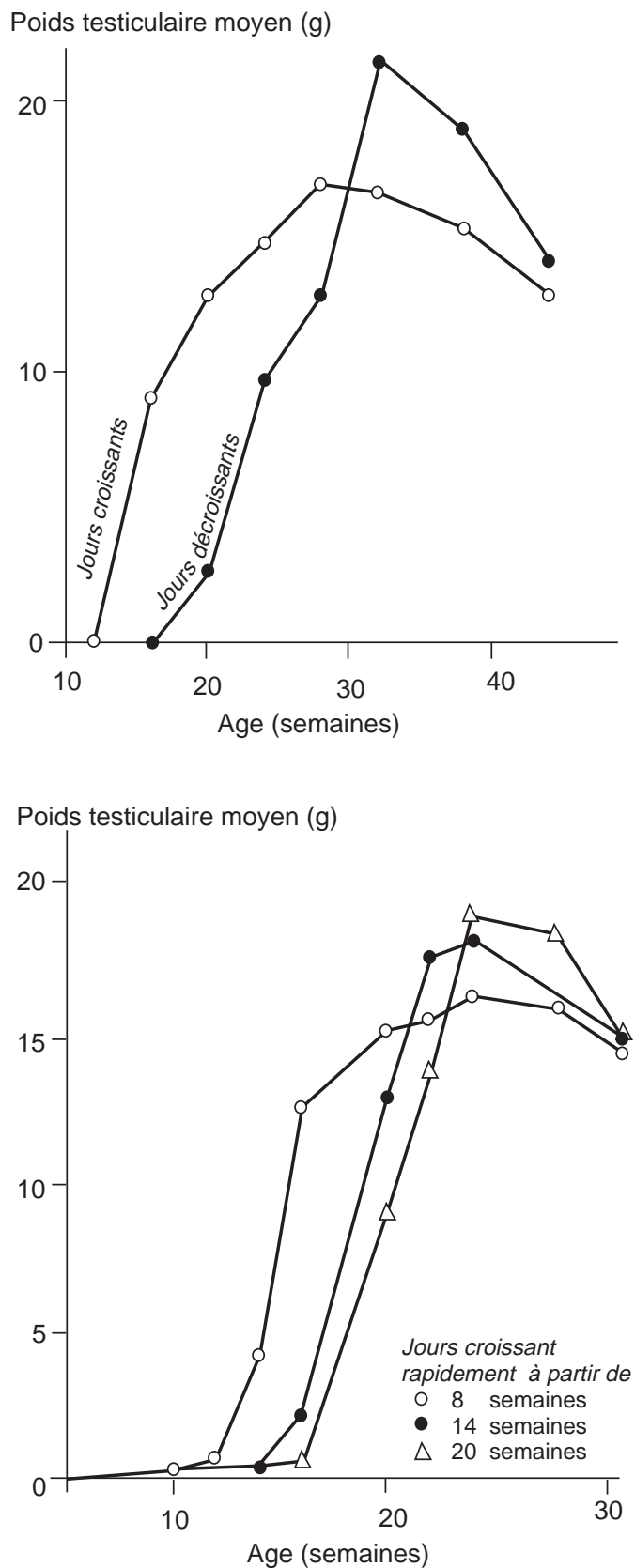
longs constants), si la photopériode journalière, de $8 \mathrm{~h}$ à la naissance, est augmentée progressivement (+20 min/sem) jusqu'à $16 \mathrm{~h}$, valeur atteinte dans ces conditions à l'âge de 24 semaines. Toutefois l'âge auquel le poids des testicules est maximum n'est guère modifié (20-24 semaines), par suite d'un ralentissement marqué de la vitesse de croissance des testicules à partir de 16 semaines d'âge.

Il est possible de rendre la maturité sexuelle nettement plus précoce si l'augmentation de la durée du jour est plus rapide $(+1$ $\mathrm{h}$ /sem). En effet le contrôle de la production spermatogénétique de coqs élevés sous $8 \mathrm{~h}$ d'éclairement journalier, et soumis à cette augmentation d'éclairement à partir de 8 semaines d'âge, nous a permis de montrer qu'ils arrivent à maturité sexuelle dès 16 semaines d'âge pour les plus précoces, ou 20 semaines chez les plus tardifs, donc un mois et plus avant les coqs élevés en jours longs constants. Ce résultat est attesté par les taux de fécondation obtenus en reproduction naturelle.

Mais des jours croissants aussi rapidement sont incapables de déclencher la phase pubère en-deçà de 12 semaines d'âge, même quand les coqs y sont soumis dès l'âge de 4 semaines : tout se passe comme s'il y avait un état photoréfractaire juvénile.

Selon nos résultats, l'avance du développement des testicules correspond à une diminution du poids qu'ils atteignent en fin de croissance (tableau 1). A l'opposé, c'est en retardant la croissance des testicules que nous avons pu obtenir les poids les plus élevés à la fin de celle-ci.

Tout se passe comme s'il y avait antagonisme entre ces deux caractères. Cela peut s'expliquer par la différenciation précoce des cellules de Sertoli en jours croissants, ce qui en diminue le nombre final. Cette différenciation est au contraire tardive en jours décroissants, ce qui prolonge la période de prolifération des cellules de Sertoli. La taille du territoire colonisable par les cellules germinales se trouve ainsi réduite ou augmentée.

Par ailleurs le feed-back plus ou moins précoce que les hormones gonadiques exercent sur le système hypothalamo-hypophysaire a probablement un rôle important dans l'antagonisme précité, mais nous n'avons pu le quantifier dans ces expériences.

Dans tous les cas, il y a régression testiculaire une fois que les jours sont devenus longs, et cette régression est d'autant plus marquée que le poids atteint par les testicules en fin de croissance est plus élevé. Un second antagonisme apparaît donc à ce niveau, sans doute par augmentation du feedback testiculaire.

$\mathrm{Au}$ total il y a donc des interactions importantes entre les périodes successives du développement testiculaire : en le rendant précoce, on en limite le niveau à l'âge adulte, mais si on augmente trop ce dernier, on le fait régresser plus vite.

\section{5 / Phase de photosensibilité maximum}

Chez les Oiseaux sauvages, le fractionnement d'une durée d'éclairement journalière courte, non gonadostimulante quand elle est donnée en une seule fois, peut conduire à une stimulation intense du développement testiculaire. Il en est de même en nycthémères de durée supérieure à $24 \mathrm{~h}$, à condition que la photopériode courte revienne tous les multiples impairs de $12 \mathrm{~h}$. A partir de ces expériences s'est peu à peu dégagé le concept de rythme circadien de la photosensibilité, idée émise par ailleurs depuis longtemps par Pfeiffer, et reprise par Bünning chez les Végétaux, puis Pittendrigh et Follett chez les Oiseaux.

La mise en évidence d'un tel rythme a depuis été faite systématiquement dans plusieurs espèces, comme par exemple chez le mâle de la Caille domestique, en utilisant deux photopériodes quotidiennes courtes, choisies de manière à ce que leur durée totale soit celle d'un jour court non gonadostimulant. La première photopériode journalière est par exemple de $6 \mathrm{~h}$, pour bien synchroniser les Oiseaux, et la seconde est choisie plus courte, pour connaître avec précision le moment où ils sont le plus photosensibles. Parfois même, il ne s'agit que d'un flash, de manière à bien marquer que l'emplacement de la seconde photopériode est plus important que sa durée.

Diverses expériences bâties sur ce modèle a priori, ont fait conclure que la seconde photopériode doit se situer environ $12 \mathrm{~h}$ après le début de la première pour qu'il y ait gonadostimulation. Mais il s'agit encore une fois d'expériences à court terme, où seul le début de la phase rapide de croissance testiculaire a été étudié. Qu'en est-il du niveau de développement final des testicules, et que devient-il pendant la vie adulte?

Tableau 1. Poids moyen maximum atteint par les testicules chez des coqs M519 soumis à des jours croissants de 8 heures à 16 heures (+ 1 heure par semaine pendant 8 semaines) à différents âges (d'après de Reviers 1975 et 1977).

\begin{tabular}{|l|c|c|c|c|}
\hline Age à la photostimulation (semaines) & $8^{(1)}$ & $14^{(1)}$ & $20^{(1)}$ & $24^{(2)}$ \\
\hline Age du démarrage de la phase rapide du développement testiculaire & 12 & 14 & 16 & 18 \\
Poids moyen maximum (g) des testicules (CV = 25\%) & 31 & 35 & 37 & 44 \\
\hline
\end{tabular}

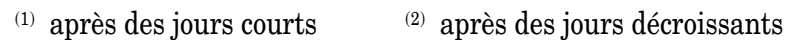


Nous avons utilisé chez le Coq une photopériode principale de $7 \mathrm{~h}$, suivie d'une photopériode secondaire de $1 \mathrm{~h}$, placée toujours à la même heure dans un lot expérimental donné, mais à des moments différents dans chaque lot respectif. Dans ces conditions, le développement testiculaire, évalué à 8,14 ou 16 semaines d'âge, est toujours plus élevé que sous $8 \mathrm{~h}$ d'éclairement quotidien données en une seule fois. Il est maximum quand la photopériode courte est donnée 16 à 18 heures après le début de la photopériode longue, soit encore 6 à 8 heures avant l'allumage de celleci (figure 4a).

Cette dernière formulation est d'ailleurs celle qui coïncide avec le rythme d'activité des coqs. Dans le cas présent, ils synchronisent leur éveil sur le début de la photopériode courte, contrairement au modèle posé a priori, et c'est donc à fin de leur journée qu'ils se trouvent recevoir les $7 \mathrm{~h}$ de lumière qui étaient supposées les synchroniser ! Cela correspond d'ailleurs à un "jour subjectif » de 13 à $15 \mathrm{~h}$, ce qui est conforme à ce qui est observé dans les autres espèces. Il aurait été de 17 à $19 \mathrm{~h}$ si le début de la photopériode de $7 \mathrm{~h}$ avait coïncidé avec le réveil.

Ce fractionnement de la photopériode a donc l'intérêt de déclencher un développement rapide et précoce des testicules, comme le font par ailleurs les jours croissants. Mais c'est encore une fois au détriment du niveau final de leur développement comme le montre la figure $4 \mathrm{~b}$, et ce fait, occulté dans la littérature scientifique, enlève beaucoup de son intérêt pratique à ce type de programme d'éclairement fractionné, si ce n'est de réduire la dépense d'électricité !

\section{6 / Les résultats sont-ils semblables chez d'autres types de coqs et d'autres espèces avicoles?}

Nous avons eu l'occasion de travailler sur d'autres lignées ou croisements de coqs, aujourd'hui disparues du marché. En jours longs, chez des coqs M41 et M33, par exemple, de type "ponte » comme les M519, et de caractéristiques très voisines quant au poids corporel, nous avons observé, contrairement à ces derniers et aux coqs de type chair I99 (figure 5), que le poids testiculaire atteint à maturité sexuelle pouvait être maintenu pendant des semaines, voire des mois.

A l'opposé, nous avons observé sous des jours courts, une diminution importante du nombre de spermatozoïdes récoltés chez des coqs adultes de type chair à croissance lente (qui sont utilisés pour la production de poulets plus âgés que le poulet industriel classique).

Il est vrai que ces coqs, assez agressifs, avaient dû être élevés sous lumière rouge de faible intensité (10 lux environ) pendant la croissance, pour subir ensuite une intensité lumineuse bien plus forte en bâtiments pour adultes (150 lux et plus). Peut-être cette augmentation de l'intensité lumineuse a-t-elle correspondu à une stimulation équivalant à des jours longs?

Les effets de l'éclairement sur le développement testiculaire sont encore mal connus chez les autres espèces avicoles. Il y a une exception, la Pintade. Le mâle de cette espèce réagit à la durée du jour de façon beaucoup plus marquée que le Coq. Sous photopériodes

\section{Le fractionnement de la durée d'éclairement permet d'accélérer et d'augmenter la croissance \\ testiculaire, mais au détriment du niveau final de développement.}

Figure 4. Mise en évidence d'une période de photosensibilité maximum vis-à-vis du développement testiculaire de coqs en croissance, jusqu'à 16 semaines d'âge (a) et au-delà (b) (d'après de Reviers 1980). Les coqs reçoivent une photopériode principale de 7 heures et une photopériode secondaire de 1 heure commençant 10 à 20 heures après allumage de la photopériode principale.

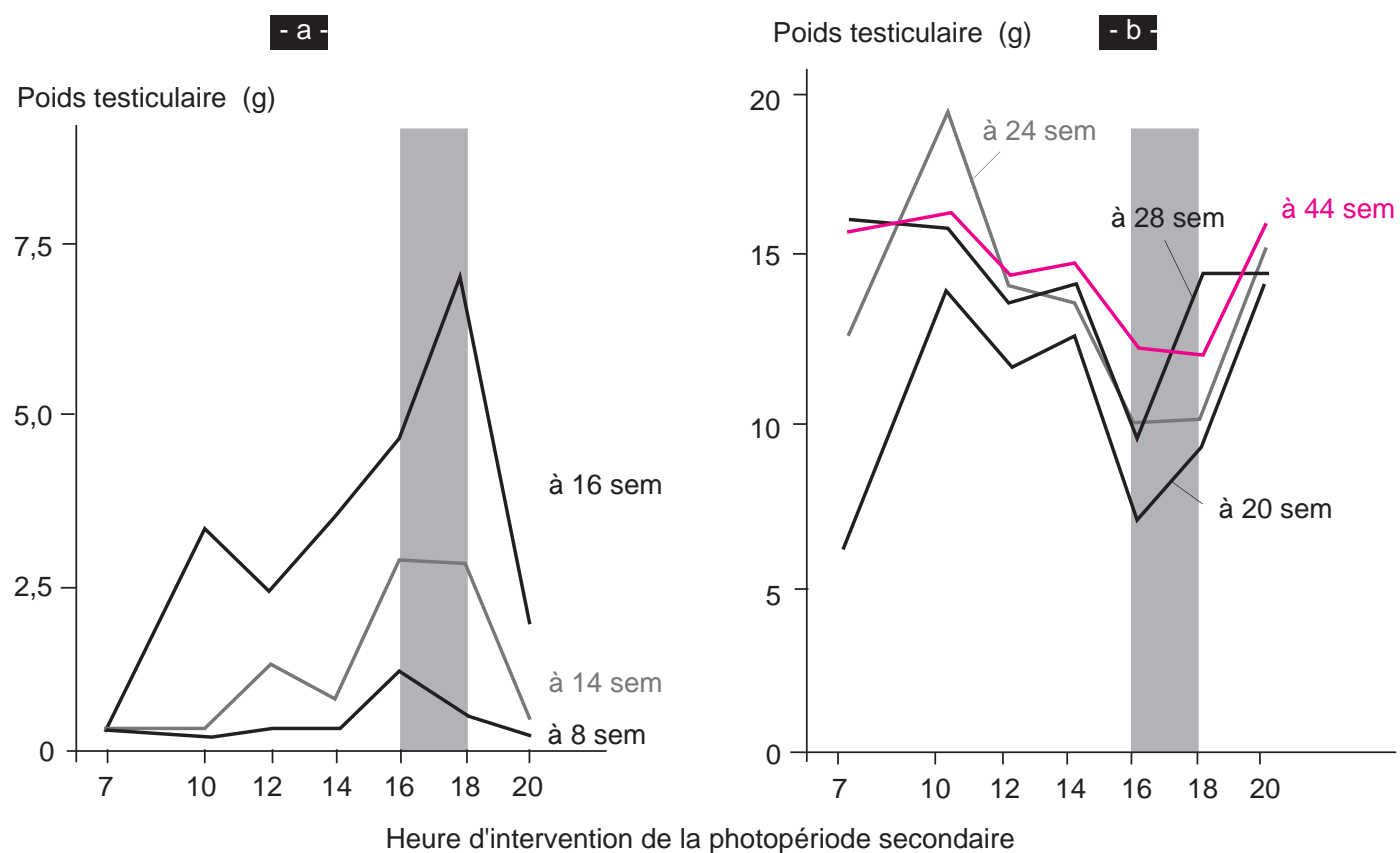


Figure 5. Développement pondéral des testicules suivant l'origine génétique des coqs, placés sous 16 h d'éclairement quotidien. M41, M55, M33 : type " chair ; 199 : type " ponte ».

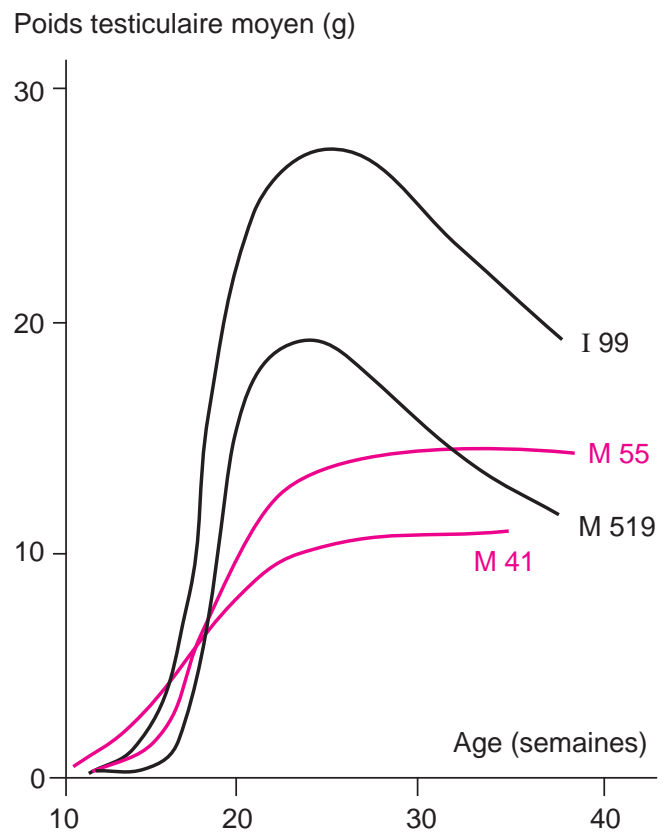

quotidiennes constantes, le développement testiculaire y est en effet très précoce en jours longs ( 14 ou $20 \mathrm{~h}$, figure 6 ), alors qu'il est fortement retardé par des jours courts $(7 \mathrm{~h})$. A cette première différence avec le Coq s'ajoute le fait que, chez la Pintade, le poids testiculaire est peu élevé à maturité sexuelle en jours longs, mais reste stable par la suite. A l'opposé, sous les jours courts, le retard de la phase pubère est suivi d'un développement spectaculaire des testicules, qui régressent rapidement par la suite.

Paradoxale par rapport au Coq, la réponse testiculaire de la Pintade ne l'est donc pas moins par rapport aux Oiseaux sauvages.

Cependant l'emploi de jours croissants chez la Pintade permet une précocité intermédiaire, d'autant meilleure que la photopériode varie plus brutalement (passage en un seul jour de 7 à $14 \mathrm{~h}$ déclairement) ou de façon plus prolongée (augmentation du jour de 7 à $20 \mathrm{~h}$ par paliers hebdomadaires de $1 \mathrm{~h}$ ). Dans ces conditions, le niveau adulte de production de spermatozoïdes est élevé, et se maintient pendant des mois. Ce type d'éclairement peut donc être considéré comme satisfaisant dans la pratique.

\section{Conclusion}

De tout ce qui précède il ressort que chez le Coq, espèce réputée peu photosensible, l'éclairement journalier permet en réalité de contrôler dans une large mesure soit la précocité du développement des testicules, soit son niveau final, soit encore, et surtout, la persistance de ce développement chez l'adulte. Mais les variations de ces trois caractéristiques qui peuvent être induites par le photopériodisme sont quelque peu antagonistes, de sorte qu'il n'est pas possible pour le moment de les porter simultanément à leur maximum.

Sauf cas particuliers qui ne concernent pas la production industrielle de poussins de chair, les jours courts sont dans l'état actuel des connaissances et de la sélection, les seuls capables de maintenir l'activité spermatogénétique à niveau élevé pendant la durée d'utilisation pratique des coqs de type chair. Mais ces jours courts ont l'inconvénient de retarder la maturité sexuelle, en particulier si l'alimentation est rationnée en vue de limiter leur état d'engraissement. Autrement dit il y a incompatibilité entre les conditions d'éclairement qui maintiennent le mieux la ponte et celles qui assurent la meilleure persistance de la production de spermatozoïdes.

Dans la pratique, cette difficulté est surmontée par l'utilisation d'un nombre très élevé de coqs (8 à $12 \%$ ) par rapport à celui des poules, ce qui masque l'insuffisance de la production de spermatozoïdes individuelle, et de bons résultats sont obtenus depuis que la tendance à l'engraissement des coqs est limitée par une alimentation séparée des poules, et rationnée.

Malgré l'efficacité de ces palliatifs, il n'en reste pas moins, comme nous l'avons montré, qu'il serait possible de réduire de trois à dix fois le nombre des coqs si l'insémination artificielle était utilisée. Cette réduction d'effectif, résultat conjoint de conditions d'élevage mieux adaptées et de l'utilisation des spermatozoïdes en fonction des besoins de la fécondation, permettrait de diminuer le prix de revient des poussins. Le surcroît de maind'œuvre occasionné par l'insémination artificielle, générateur de nombreux emplois, peut en effet être plus que compensé par l'écono-

Figure 6. Variations du développement testiculaire induites par la durée d'éclairement chez la Pintade (Brillard et de Reviers 1981).

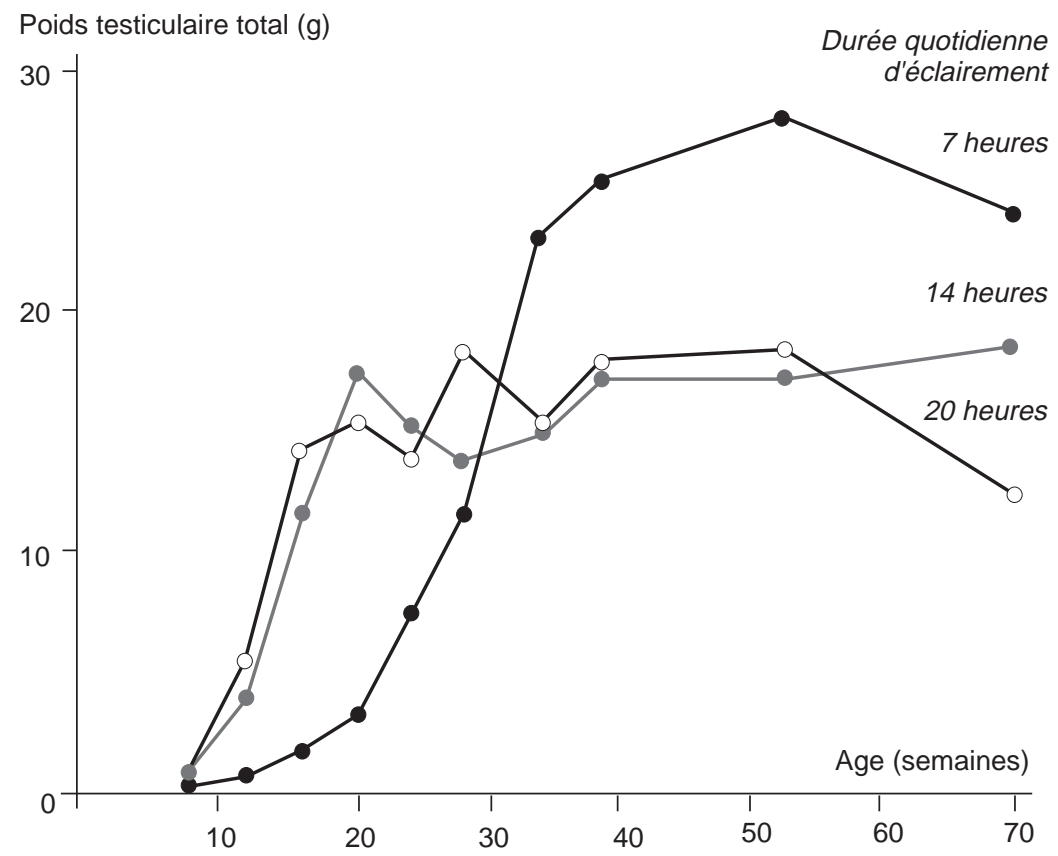

\section{Chez la pintade mâle, les jours longs accélèrent la croissance testiculaire, jusqu'à un poids plus faible qu'en jours courts, mais stable.}


mie d'aliment faite sur les coqs surtout dans les pays où la main-d'œuvre est bon marché. En outre, la réduction du nombre de coqs peut être faite au bénéfice des plus aptes à la croissance, d'où de meilleures performances de la descendance. Encore faut-il que le producteur de poussins y trouve bénéfice en les vendant plus cher à l'état de poussins ou de poulets de chair, chose difficile quand l'éleveur est le client de l'accouveur! Par ailleurs l'insémination artificielle a notamment pour inconvénients d'être routinière, exigeante en technicité, et de transférer sur des hommes une responsabilité jusque là imputable aux seuls coqs. Elle rend donc dépendante de la qualité du personnel.

\section{Références bibliographiques}

Brillard J.P., de Reviers M., 1981. Testis development and daily sperm output in guinea-fowl raised under constant daily photoperiods. Reprod. Nutr. Develop., 21, 1105-1112.

Brillard J.P., de Reviers M., 1989. L'insémination artificielle chez la Poule. Bases physiologiques et maintien du taux de fécondation des œufs. INRA Prod. Anim., 2 (3), 197-203.

Bünning E., 1936. Die endogene Tagesrhytmik als Grundlage der Photoperiodischen Reaktion. Ber. Deut. Bot., 54, 590-607.

Farner D.S., 1958. Photoperiodism in animals with special reference to avian testicular cycles. Proc. 9th. Annual Biol. Coll., Oregon State College, 17-29.

Hamner W.M., 1966. Photoperiodic control of the annual testicular cycle in the house finch, Carpodacus mexicanus. Gen. Comp. Endocrinol., 7, 224-233.

Menaker M., Eskin A., 1967. Circadian clock in photoperiodic time measurement. A test of the Bünning hypothesis. Science, 157 (3793), 1182-1184.

Nalbandov A.V., 1967. Endocrine background of light action. In : La photorégulation de la Reproduction chez les Oiseaux, Colloque CNRS, Montpellier, 29-52.

de Reviers M., 1988. Facteurs de variation du développement testiculaire et de la production de sper- matozoïdes. In : B. Sauveur (ed), Reproduction des Volailles et Production d'œufs, 183-208. INRA Editions, Paris.

de Reviers M., 1990. Effets du rationnement alimentaire chez le Coq de type chair. Interactions avec la durée quotidienne d'éclairement. INRA Prod. Anim., 3 (1), 21-30.

Saeki Y., 1963. Fertilizing ability of cock spermatozoa first ejaculated and changes in semen quality with age of the cock. Jap. J. Zoot. Sci., 34, 111-125.

Schwab R.G., 1970. Light-induced prolongation of spermatogenesis in the european starling, Sturnus vulgaris. The Condor, 72, 466-470.

Tanaka K., Mather F.B., Wilson W.O., McFarland L.Z., 1965. Effects of photoperiods on early growth of gonads and on potency of gonadotrophins of the anterior pituitary in Coturnix. Poult. Sci., 44, 662665 .

Williams J., de Reviers M., 1981. Variations in the plasma level of luteinizing hormone and androstenedione and their relationship with the adult daily sperm output in cockerels raised under different photoschedules. Reprod Nutr. Develop., 21, 1125-1135.

Wolfson A., 1966. Environmental and neurondocrine regulation of annual gonadal cycles and migratory behavior in birds. Rec. Prog. Horm. Res., 22, 177-244.

\section{Abstract}

Photoperiodicity, testicle development and sperm production in domestic fowl.

The reproductive capabilities of the cockerel are strongly influenced by daylength. Its variations strongly influence the age at which testicular development occurs, and consequently the age at sexual maturity. Both are advanced by increasing the daily photoperiods in growing cockerels. However the sperm production markedly declines after sexual maturity in cockerels submitted to long days, though they are known to allow high egg laying rates in the hen. By contrast, the adult sperm production may be well maintained under short constant days (e.g. 8L :16D), which induce delay in sexual maturity. Therefore there is an antagonism between photoschedules required to reach precocious sexual maturity and those able to maintain sperm production.

In the industry, adult broiler cockerels are raised under long days, together with the hens, to reproduce by natural mating. Many males (8 to 12 per
100 females) are used to compensate for the decline in their adult sperm production. They are furthermore submitted to restricted feeding, to prevent them from fattening and to stimulate their libido. However a number of deficient cockerels must often be replaced during the reproductive period. On an experimental basis, artificial insemination leads to a better management of males, through the use of specific photoschedules. It also permits the number of males to be reduced markedly by optimizing the use of semen to match the fertility requirements of hens. But artificial insemination is expensive and its technical requirements are rather hard to meet. It is therefore not in use for broiler production in countries where the cost of labour is high, even though it maximises profits in other domestic bird species.

de REVIERS M., 1996. Photopériodisme, développement testiculaire et production de spermatozoïdes chez les oiseaux domestiques. INRA Prod. Anim., 9 (1), 35-44. 